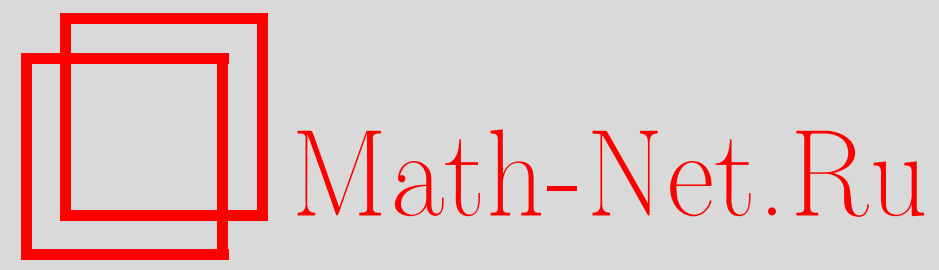

R. Norvaiša, Strassen's law for weighted sup-norms, Теория вероятн. и ее примен., 1997, том 42, выпуск 4, 783-795

DOI: https://doi.org/10.4213/tvp2596

Использование Общероссийского математического портала Math-Net.Ru подразумевает, что вы прочитали и согласны с пользовательским соглашением http://www . mathnet.ru/rus/agreement

Параметры загрузки:

IP : 54.166 .219 .16

26 апреля 2023 г., 08:02:01

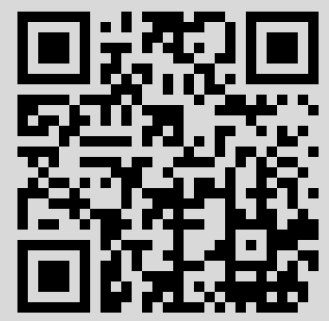


(c) $1997 \Gamma$.

NORVAIŠA R.

\section{STRASSEN'S LAW FOR WEIGHTED SUP-NORMS ${ }^{1)}$}

Пусть $S$ - процесс частичных сумм, построенный по последовательности независимых одинаково распределенных случайных величин со средним 0 и дисперсией 1 . Доказано, что последовательность $\left\{(2 n L L n)^{-1 / 2} S(n \cdot) ; n \geqslant 1\right\}$ подчиняется функциональному закону повторного логарифма относительно взвешенной sup-нормы в том случае, если предельное множество компактно.

Ключевые слова и фразы: закон повторного логарифма в форме Штрассена, процесс частичных сумм, множество Штрассена.

1. Introduction. Suppose that $X_{1}, X_{2}, \ldots$ is a sequence of independent identically distributed (i.i.d.). random variables with mean zero and variance 1 defined on a common probability space $(\Omega, \mathcal{A}, \mathbf{P})$. Let $S=$ $\{S(t): t \geqslant 0\}$ be a partial sum process based on $\left\{X_{i}: i \geqslant 1\right\}$, i.e., $S(t):=$ $\sum_{i=1}^{[t]} X_{i}$ for $t \geqslant 1$ and $S(t):=0$ for $0 \leqslant t<1$, where $[r]$ denotes the integer part of $r$. Let $S^{c}=\left\{S^{c}(t): t \geqslant 0\right\}$ denote a continuous version of $S$, that is the stochastic process obtained by linearly interpolating 0 and $\sum_{i=1}^{k} X_{i}$ at $k$ for $k=1,2, \ldots$. For each integer $n \geqslant 1$, define a scaled partial sum process

$$
S_{n}=\left\{S_{n}(t): t \geqslant 0\right\}
$$

by

$$
S_{n}(t):=\frac{S(n t)}{b_{n}} \quad \text { and } \quad b_{u}:=b(u):=\sqrt{2 u L L u}, \text { for } u \geqslant 0,
$$

where $L u:=\log (u \vee e)$ for $u \geqslant 0$. Similarly there is defined a sequence $\left\{S_{n}^{c}: n \geqslant 1\right\}$. Then each $S_{n}^{c}$ has sample paths in the space $\mathbb{C}([0, \infty))$ of continuous functions on $[0, \infty)$ endowed with the topology of uniform convergence on compact intervals. Strassen's law of the iterated logarithm asserts that, with probability one, the sequence $\left\{S_{n}^{c}: n \geqslant 1\right\}$ is convergent and clusters the set $\mathcal{S}$ in $\mathbb{C}([0, \infty))$, where

$$
\mathcal{S}=\left\{\varphi(t)=\int_{0}^{t} \psi(u) d u, t \geqslant 0: \int_{0}^{\infty} \psi^{2}(u) d u \leqslant 1\right\}
$$

*Institute of Mathematics and Informatics, Akademijos, 4, Vilnius 2600, Lithuania.

${ }^{1)}$ Supported by Deutsche Forchungsgemeinschaft, Sonderforschungsbereich 343 «Diskrete Strukturen in der Mathematik», at the University of Bielefeld, Germany. 
To extend this result to the case of uniform convergence on a whole half line, Wichura [16] used a weighted sup-norm $\|f\|_{w}:=\sup _{t}|f(t)| w(t)$ with

$$
w(t)=\frac{1}{b}(t \vee 3) \text { for } t \geqslant 0 .
$$

Namely, for such a function $w$, we have

$$
\left\{\begin{array}{l}
\mathbf{P}\left\{\left\{\lim _{n \rightarrow \infty} \inf _{\varphi \in \mathcal{S}}\left\|S_{n}^{c}-\varphi\right\|_{w}=0\right\}\right\}=1, \\
\forall \varphi \in \mathcal{S}, \quad \mathbf{P}\left\{\left\{\liminf _{n \rightarrow \infty}\left\|S_{n}^{c}-\varphi\right\|_{w}=0\right\}\right\}=1 .
\end{array}\right.
$$

We are seeking for a description of the class of all functions $w$ satisfying (1.2). The question is how fast the function $w$ can decrease to zero near infinity and/or increase to infinity near zero with (1.2) still holding true. This is of interest because statement (1.2) holding with such a function $w$ permits an enlargement of the class of continuous functions for which a continuous mapping theorem holds true (cf, Section 3 in [14]). Using classical law of the iterated logarithm one can show that the function (1.1) can not be replaced in (1.2) by a function $w$ such that $w(t) b(t \vee 1) \longrightarrow 0$ as $t \rightarrow \infty$. However, the question of characterizing the behaviour of $w$ near zero satisfying (1.2) is open. The aim of the present paper is to answer this question.

It is convenient to exclude zero from the domain of definition of the function $w$ when its behaviour near zero is treated. More generally, we will consider functions defined on a locally compact space $T$. So, typical examples of $T$ are intervals $(0, \infty),[0,1)$ and $[1, \infty)$. A positive function $w$ on $T$ is called a weight function on $T$ if it is bounded on every compact subset of $T$. Given a weight function $w$ on $T$, as a sample path space, we consider a Banach space $\left(\mathrm{B}(T, w),\|\cdot\|_{w}\right)$, where

$$
\mathbb{B}(T, w):=\left\{f \in \mathbb{R}^{T}:\|f\|_{w}=\sup _{t \in T}|f(t)| w(t)<\infty\right\}
$$

and $\mathbb{R}^{T}$ denotes the class of all real valued functions on $T$. Denote by $C_{0}((0, \infty), w)$ the subspace of $\mathbb{B}_{0}((0, \infty), w)$ consisting of continuous functions $f$ such that $f w$ vanishes at zero and infinity. The identity function on $T$ and the indicator function of a set $A$ will be denoted by $I$ and $\mathbb{I}_{A}$, respectively. We will make no notational distinction between functions (and processes) defined on $(0, \infty)$ (or on $[0, \infty)$ ) and their restrictions to $(0,1]$ or to $[1, \infty)$. The restrictions will be clear from the context.

Turning to the question of what the possible weight functions could be, first note that $\|\varphi\|_{w}<\infty$ for all $\varphi \in \mathcal{S}$ whenever $\sup _{t>0} \sqrt{t} w(t)<\infty$. Next, we demonstrate that Strassen's law ceases to hold for the weight function $w_{0}$ defined by $w_{0}(t):=t^{-1 / 2}$ for $t \in(0,1]$. Let $Y_{1}, Y_{2}, \ldots$ be independent 
$\mathcal{N}(0,1)$ identically distributed random variables and let $\{V(t): t \geqslant 0\}$ be a Gaussian process with a covariance function

$$
\mathrm{E} V(t) V(s)=\exp \left\{-\frac{|t-s|}{2}\right\} \text { for } t, s \in(0, \infty)
$$

Using a time change, by the result of Nisio [13], it follows that

$$
\begin{aligned}
& \lim _{n \rightarrow \infty}(2 n L L n)^{-1 / 2} \sup _{0<t \leqslant 1}\left|\sum_{i=1}^{[n t]} Y_{i}\right| w_{0}(t) \\
& =\lim _{T \rightarrow \infty}(2 L T)^{-1 / 2} \sup _{0<t \leqslant T}|V(t)|=1 \quad \text { a.s. }
\end{aligned}
$$

Then the arguments of Lai $[10$, p. 8$]$ assure that the set of limit points of the sequence

$$
\left\{(2 n L L n)^{-1 / 2} \sum_{i=1}^{[n \cdot]} Y_{i}: n \geqslant 1\right\}
$$

in the Banach space $\left(\mathbf{B}\left((0,1], w_{0}\right),\|\cdot\|_{w_{0}}\right)$ is empty.

The main result of this paper is twofold. First, the Strassen set $\mathcal{S}$ is compact in $\mathbb{C}_{0}((0, \infty), w)$ if and only if $\sqrt{I} \in \mathbb{C}_{0}((0, \infty), w)$ that is

$$
\lim _{t \downarrow 0, t \uparrow \infty} \sqrt{t} w(t)=0
$$

(cf. Corollary to Proposition below). Second, if (1.3) holds (when restricted to $(0,1])$ then the sequence $\left\{S_{n}: n \geqslant 1\right\}$ converges to and clusters the set $\mathcal{S}$ in $\left(\mathbb{B}((0,1], w),\|\cdot\|_{w}\right)$. The sequence of discontinuous scaled partial sum processes in the second statement can be replaced by a sequence $\left\{S_{n}^{c}: n \geqslant 1\right\}$.

To prove the convergence result, we first derive an analogous result for a truncated Brownian motion using ideas of Strassen [14], Deuschel and Stroock [7] and Baldi et al. [2] (cf. Theorem below). Then, (1.2) for a partial sum process, follows via Skorokhod embedding just as in [14] (cf. Corollary to Theorem below). We note that condition (1.3) does not guarantee the existence of Wiener measure as a Radon probability measure on a Borel $\sigma$ algebra of $C_{0}((0, \infty), w)$ (cf. the end of the next section for more details). Additional argument supporting the last claim follows from results of Beibel and Lerche [3]. Therefore, the recent results of Ben Arous and Ledoux [4] and of Deheuvels and Lifshits [6], concerning the Strassen law for a Brownian motion, are not applicable in this case.

Finally, we note that conditions on a weight function for Strassen's law to hold for a uniform empirical process are different than those given in Corollary to Theorem below. Indeed, let $w$ be a weight function on $(0,1)$. Set

$$
\bar{w}(t):=\sup \left\{w(s): s \in\left[t, \frac{1}{2}\right] \text { or }\left[\frac{1}{2}, t\right]\right\} .
$$

5 Теория вероятностей и ее применення, № 4 
Let $\left\{U_{i}: i \geqslant 1\right\}$ be a sequence of i.i.d. uniform random variables. Then, by Theorem 3.2 of Alexander and Talagrand [1], a sequence $\left\{b_{n}^{-1} \sum_{i=1}^{n}\left(\mathbb{I}_{\left[U_{i}, 1\right]}-\right.\right.$ $I): n \geqslant 1\}$ satisfies Strassen's law in $\mathrm{B}((0,1), w)$ if and only if

$$
\lim _{t \downarrow 0, t \uparrow 1} \sqrt{t(1-t)} w(t)=0 \quad \text { and } \quad \int_{0}^{1} \frac{\bar{w}^{2}(t) d t}{L L(1 /(t(1-t)))}<\infty .
$$

2. The Strassen set. In this section, we give a necessary and sufficient condition on a weight function $w$ for the Strassen set $\mathcal{S}$ to be compact in $\mathbb{C}_{0}((0, \infty), w)$.

Let $T$ be a locally compact space and let $w$ be a weight function on $T$. Denoting by $\mathrm{C}(T)$ the set of all real valued continuous functions on $T$, set

$$
\begin{gathered}
\mathbb{C}_{0}(T, w):=\{f \in \mathbb{C}(T): \forall \varepsilon>0, \exists \text { a compact } K \text { in } T \text { such that } \\
|f(t)| w(t)<\varepsilon, \forall t \in T \backslash K\} .
\end{gathered}
$$

Then, equipped with the weighted sup-norm $\|\cdot\|_{w}, C_{0}(T, w)$ is a separable real Banach space. Usually, in analysis (cf. [12]), the weight function $w$ is defined to be upper-semicontinuous which is stronger property than just local boundedness. In that case, each subset $A(f, \varepsilon):=\{t \in T:|f(t)| w(t) \geqslant \varepsilon\}$ is closed in $T$ and the space $C_{0}(T, w)$ consists of those $f \in \mathbb{C}(T)$ for which $A(f, \varepsilon)$ is compact for every $\varepsilon>0$.

The following statement is an extension of the classical Arzelà-Ascoli theorem for the Banach space $C_{0}(T, w)$. We sketch the proof of this known result because it is crucial for describing compactness of the Strassen set $\mathcal{S}$.

Lemma. Let $T$ be a locally compact set, and let $w$ be a weight function on $T$. A subset $\mathcal{F}$ of $\mathcal{C}_{0}(T, w)$ is totally bounded if and only if

(1) $\mathcal{F}$ is bounded that is $\sup _{f \in \mathcal{F}}\|f\|_{w}<\infty$;

(2) $\mathcal{F}$ is equicontinuous that is for each $t \in T$ and for every $\varepsilon>0$ there is an open neighborhood $U_{t}$ of $t$ such that $|f(t)-f(s)|<\varepsilon$ for all $s \in U_{t}$ and for all $f \in \mathcal{F}$;

(3) for every $\varepsilon>0$ there is a compact subset $K$ of $T$ such that $|f(t)| \times$ $w(t)<\varepsilon$ for all $f \in \mathcal{F}$ and for all $t \in T \backslash K$.

$\mathrm{P}$ r o of. It is easy to see that (1), (2) and (3) are satisfied if the set $\mathcal{F}$ is totally bounded. Conversely, assume that (1), (2) and (3) hold true. To construct an $\varepsilon$-net of $\mathcal{F}$ fix an arbitrary $\varepsilon>0$. By (3), there is a compact subset $K$ of $T$ such that $|f(t)| w(t)<\varepsilon / 2$ for all $f \in \mathcal{F}$ and for all $t \in T \backslash K$. Put $w_{K}:=\sup _{t \in K} w(t)$. By (2), for each $t \in K$ there is an open neighborhood $U_{t}$ of $t$ such that $|f(t)-f(s)|<\varepsilon /\left(4 w_{K}\right)$ for all $f \in \mathcal{F}$ and for all $s \in U_{t}$. Since $K$ is compact, there are points $t_{1}, \ldots, t_{k}$ in $K$ such that $K \subset \cup_{j=1}^{k} U_{t_{j}}$. By (1), there is a finite number $A$ such that $\left|f\left(t_{j}\right)\right| \leqslant A$ for all $f \in \mathcal{F}$ and for each $j=1, \ldots, k$. Let $\left\{\alpha_{1}, \ldots, \alpha_{l}\right\}$ be an $\left(\varepsilon /\left(4 w_{K}\right)\right)$-net of the interval $[-A, A]$. Consider the (finite) collection $\Phi$ of those functions 
$\varphi$ from $\{1, \ldots, k\}$ into $\{1, \ldots, l\}$ for which the set

$$
\mathcal{F}_{\varphi}:=\left\{f \in \mathcal{F}:\left|f\left(t_{i}\right)-\alpha_{\varphi(i)}\right|<\frac{\varepsilon}{4 w_{K}}, i=1, \ldots, k\right\}
$$

is nonempty. We note that $\mathcal{F} \subset \cup_{\varphi \in \Phi} \mathcal{F}_{\varphi}$ and that $\operatorname{diam} \mathcal{F}_{\varphi} \leqslant \varepsilon$. Take any function $f_{\varphi}$ in $\mathcal{F}_{\varphi}$. Then the set $\left\{f_{\varphi}: \varphi \in \Phi\right\}$ is the $\varepsilon$-net of $\mathcal{F}$.

Let $B$ be a Banach space with a norm $\|\cdot\|_{B}, B^{*}$ be its topological dual, and let $\langle\cdot, \cdot\rangle$ be a duality between $B$ and $\mathbf{B}^{*}$. Consider a function $a$ from $T$ into $\mathbb{B}^{*}$ such that, for all $\boldsymbol{x} \in \mathbf{B}$,

$$
T \ni t \mapsto\langle x, a(t)\rangle \in \mathbb{C}_{0}(T, w)
$$

and

$$
\sup _{t \in T}\|a(t)\|_{B^{*}} w(t)<\infty
$$

Then, such a function $a$ defines a bounded operator $A$ from $B$ into $C_{0}(T, w)$ by

$$
A x:=\langle x, a(\cdot)\rangle, \quad x \in \mathbf{B},
$$

with the operator norm

$$
\|A\|=\sup _{\|x\|_{B} \leqslant 1} \sup _{t \in T}|\langle x, a(t)\rangle| w(t)=\sup _{t \in T}\|a(t)\|_{B^{*}} w(t) .
$$

The following statement is an extension of a part of Theorem VI.7.1 in [8] to the space $\mathbb{C}_{0}(T, w)$.

Proposition. Let $w$ be a weight function on a locally compact space $T$, and let $\mathrm{B}$ be a Banach space. Consider a bounded operator $A$ from $\mathrm{B}$ into $\mathbb{C}_{0}(T, w)$ defined by (2.1). Then $A$ is compact if and only if

(1) the function $a: T \mapsto B^{*}$ is (norm) continuous;

(2) the function $T \ni t \mapsto\|a(t)\|_{B^{*}} \in \mathbb{C}_{0}(T, w)$.

If, in addition, $\mathbb{B}$ is reflexive then the image of the unit ball under the compact operator $A$ is closed for the topology of the weighted sup-norm $\|\cdot\|_{w}$.

$\mathrm{P}$ r o of. Let $A$ be compact. Then the set $\left\{A x:\|x\|_{B} \leqslant 1\right\}$ is totally bounded in $\mathrm{C}_{0}(T, w)$. By (2) of Lemma, for all $\varepsilon>0$ and for every $t \in T$ there is a neighborhood $U_{t}$ of $t$ such that

$$
\|a(t)-a(s)\|_{B^{*}}=\sup _{\|x\|_{B} \leqslant 1}|A x(t)-A x(s)|<\varepsilon, \quad \forall s \in U_{t} .
$$

This implies (1) and the continuity of the function $T \ni t \rightarrow\|a(t)\|_{B^{*}}$. Moreover, by (3) of Lemma, for every $\varepsilon>0$, there exists a compact subset $K$ of $T$. such that

$$
\|a(t)\|_{B^{*}} w(t)=\sup _{\|x\|_{B} \leqslant 1}|A x(t)| w(t)<\varepsilon, \quad \forall t \in T \backslash K
$$

This proves (2) and therefore the «only if» part of the statement. 
Conversely, assume that (1) and (2) hold true. We have to show that the set $\left\{A x:\|x\|_{B} \leqslant 1\right\}$ is totally bounded in $\mathbb{C}_{0}(T, w)$. But, using (2.2) and (2.3), this is an easy consequence of Lemma.

The last statement of Proposition is a consequence of complete continuity of a compact operator and of weak compactness of a ball of a reflexive Banach space.

Consider a separable Gaussian process $G=\{G(t): t \in T\}$ with a covariance function $r$. By the Aronszajn-Kolmogorov theorem (cf. [5]), there exists a separable Hilbert space $H$ with a scalar product $[\cdot, \cdot]$ and a map $a: T \rightarrow H$ such that

$$
r(t, s)=[a(t), a(s)], \quad(t, s) \in T \times T .
$$

By Proposition, the set

$$
\mathcal{S}_{G}:=\left\{[h, a(\cdot)]:\|h\|_{H} \leqslant 1\right\}
$$

is compact in $\mathbb{C}_{0}(T, w)$ if and only if the map $a$ is continuous and the function

$$
T \ni t \mapsto \sqrt{r(t, t)} \in \mathbb{C}_{0}(T, w) .
$$

Now, we will apply these constructions when $G$ is a Brownian motion $W$, that is when $r(t, s)=t \wedge s$ for $t, s \geqslant 0$. Let $T$ be a locally compact subspace of $(0, \infty)$. For example, $T$ may be taken to be $(0, \infty),(0,1]$ or $[1, \infty)$. Denoting by $\lambda$ Lebesgue measure on $T$, consider the function $a$ from $T$ into $B^{*}=$ $\mathrm{L}_{2}(T, \lambda)$ defined by

$$
a(t)(u):=\mathbb{I}_{(0, t]}(u), \quad t, u \in T .
$$

Hence, by Proposition, for the operator $A$ defined by (2.1) and (2.4) and acting from $\mathbf{B}=L_{2}(T, \lambda)$ into $C_{0}(T, w)$ we have.

Corollary. Let $w$ be a weight function on a locally compact subspace $T$ of $(0, \infty)$. The Strassen set $\mathcal{S}$ defined is compact in $\mathbb{C}_{0}(T, w)$ if and only if the function

$$
T \ni t \mapsto \sqrt{t} \in \mathbb{C}_{0}(T, w) .
$$

The rest of this section concerns some properties of a distribution of a Brownian motion induced on the path space $C_{0}(T, w)$ satisfying (2.5). Denote by $\mathrm{M}(T)$ the set of all signed regulated Borel measures on a locally compact space $T$. For a given Borel measurable weight function $w$ on $T$, define the Banach space

$$
\mathrm{M}(T, w):=\left\{\mu \in \mathbb{M}(T):\|\mu\|_{w}:=\int_{T} \frac{d|\mu|}{w}<\infty\right\},
$$

where $|\mu|$ denotes the variation measure associated with $\mu$. If, in addition, $w$ is continuous then it is easy to show that the topological dual $\mathbb{C}_{0}(T, w)^{*}$ of 
$\mathrm{C}_{0}(T, w)$ is isometrically isomorphic to $M(T, w)$. Let $w$ be a weight function on $T$ satisfying (2.5). Then the map $A$ given by (2.1) and (2.4) is an injective bounded operator from $\mathrm{L}_{2}(T, \lambda)$ to $\mathrm{C}_{0}(T, w)$. The adjoint $A^{*}$ of $A$ is a bounded operator from $M(T, w)$ into $\mathrm{L}_{2}(T, \lambda)$ given by

$$
A^{*}(\mu)(u)=\int_{T} a(t)(u) \mu(d t)=\mu((u, \infty) \cap T) \quad \text { for } u \in T .
$$

Hence the integral

$\int_{T}[\mu((u, \infty) \cap T)]^{2} d u=\int_{T} \int_{T} s \wedge t \mu(d s) \mu(d t)<\infty, \quad$ for all $\mu \in \mathbf{M}(T, w)$.

Let $J$ be a stochastic integral operator from $\mathbf{L}_{2}(T, \lambda)$ into the space $\mathrm{L}_{0}(\Omega, P)$ of random variables with finite second moment on a probability space $(\Omega, P)$, induced by a zero mean Gaussian random measure $H$ with control measure $\lambda$ on $T$. Therefore, one can define a linear random functional $L$ :

$$
\mathrm{M}(T, w) \longrightarrow \mathrm{L}_{2}(\Omega, P)
$$

by

$$
L \mu:=J \circ A^{*}(\mu)=\int_{T} \mu((u, \infty) \cap T) H(d u), \quad \mu \in \mathrm{M}(T, w),
$$

with the characteristic function

$$
E e^{i L \mu}=\exp \left\{-\frac{1}{2} \int_{T}[\mu((u, \infty) \cap T)]^{2} d u\right\}, \quad \mu \in M(T, w) .
$$

The linear random functional $L$ defines a cylindrical probability measure $\mathcal{W}$ on $\mathrm{C}_{0}(T, w)$ corresponding to a Brownian motion $W$ (a cylindrical Wiener measure). One can infer even more in this case. Using Theorem 4.1 of [15] and the above Proposition, one can show that a cylindrical probability measure $\mathcal{W}$ is scalarly concentrated on the family of all compact circled subsets of $C_{0}(T, w)$. However, $(2.5)$ is not sufficient for a cylindrical probability measure $\mathcal{W}$ to be extendible to a (Radon) probability measure on a Borel $\sigma$-algebra of $\mathbb{C}_{0}(T, w)$ which is usual Wiener measure. In the case when the weight function $w$ is monotone, a neeessary and sufficient condition for this follows from the well-known result due to Khintchine [9]. Namely, for a Brownian motion $W$, we have

$$
\lim _{t \downarrow 0, t \uparrow \infty}|W(t)| w(t)=0 \quad \text { a.s. . }
$$

if and only if

$$
\int_{0}^{\infty} t^{-1} \exp \left\{-\frac{c}{t w^{2}(t)}\right\} d t<\infty \text { for all } c>0 .
$$


A typical example of a weight function satisfying (2.6) is a function $w$ such that

$$
\lim _{t \downarrow 0, t \uparrow \infty} \sqrt{t L L\left(t \vee \frac{1}{t}\right)} w(t)=0 .
$$

3. Strassen's theorem. Let $w$ be a weight function on a locally compact space $T$. Then $\mathrm{C}_{0}(T, w)$ is a closed subspace of the Banach space $\left(\mathbf{B}(T, w),\|\cdot\|_{w}\right)$. For any subset $\mathcal{F} \subset \mathbf{B}(T, w)$ and for any function $f \in$ $\mathbf{B}(T, w)$, put

$$
d_{w}(f, \mathcal{F}):=\inf _{\varphi \in \mathcal{F}}\|f-\varphi\|_{w} .
$$

For each sequence $\left\{f_{n}: n \geqslant 1\right\} \subset \mathrm{B}(T, w)$, set

$$
C_{w}\left(\left\{f_{n}: n \geqslant 1\right\}\right):=\left\{f \in \mathbf{B}(T, w): \liminf _{n \rightarrow \infty}\left\|f_{n}-f\right\|_{w}=0\right\} .
$$

We wish to consider a weighted Brownian motion $w W$ under the condition (1.3). However, as it was noted earlier, the supremum of $w W$ over $(0, \infty)$ is infinite. Therefore, for each integer $n \geqslant 1$, we define a scaled and truncated Brownian motion $\widetilde{Z}_{n}=\left\{\widetilde{Z}_{n}(t): t \in T\right\}$ by

$$
\widetilde{Z}_{n}(t):=Z_{n}(t) \mathbb{I}_{\Delta_{n}}(t)= \begin{cases}\frac{W(n t)}{b_{n}} & \text { if } t \in \Delta_{n}, \\ 0 & \text { if } t \in T \backslash \Delta_{n},\end{cases}
$$

where $\Delta_{n}:=[1 / n, n]$ and $b_{n}:=\sqrt{2 n L L n}$.

We start by proving the Strassen law of the iterated logarithm in $\mathrm{B}(T, w)$ for the sequence $\left\{\widetilde{Z}_{n}: n \geqslant 1\right\}$.

Theorem. Let $T$ be any of the intervals $(0, \infty),(0,1]$ or $[1, \infty)$ and let $w$ be a weight function on $T$. If (2.5) holds then

$$
\lim _{n \rightarrow \infty} d_{w}\left(\widetilde{Z}_{n}, \mathcal{S}\right)=0 \quad \text { a.s. }
$$

and

$$
C_{w}\left(\left\{\widetilde{Z}_{n}: n \geqslant 1\right\}\right)=\mathcal{S} \text { a.s. }
$$

$\mathrm{R} \mathrm{e} \mathrm{m}$ a $\mathrm{rk}$. In the case when $T=(0, \infty)$, condition (2.5) is equivalent to the relation (1.3).

P r o o f. We prove Theorem when $T=(0, \infty)$ and note that the other cases can be proved analogously. We begin by proving (3.1). For any integer $m$, put $w^{m}:=w \mathbb{I}_{\Delta_{m}}$. Then, for each $m$ and for each $n \geqslant m$, we have

$$
d_{w}\left(\widetilde{Z}_{n}, \mathcal{S}\right) \leqslant d_{w^{m}}\left(\widetilde{Z}_{n}, \mathcal{S}\right)+\left\|\widetilde{Z}_{n} \mathbb{I}_{T \backslash \Delta_{m}}\right\|_{w}+\sup _{\varphi \in \mathcal{S}}\left\|\varphi \mathbb{I}_{T \backslash \Delta_{m}}\right\|_{w} .
$$


Let $\left\{\delta_{n}: n \geqslant 1\right\}$ be a sequence of real numbers such that $\delta_{n} \rightarrow 0$ and $n \delta_{n} \rightarrow \infty$, as $n \rightarrow \infty$. Then, for each $n$ and $m$ such that $\delta_{n}<1 / m$, we have

$$
\begin{aligned}
\left\|\tilde{Z}_{n} \mathbb{I}_{(0,1 / m)}\right\|_{w} \leqslant & \sup _{1 / n \leqslant t \leqslant \delta_{n}} \sqrt{t} w(t) \sup _{1 \leqslant s<\infty} \frac{|W(s)|}{\sqrt{2 s L L s}} \\
& +\sup _{\delta_{n} \leqslant t \leqslant 1 / m} \sqrt{t} w(t) \sup _{n \delta_{n} \leqslant s<\infty} \frac{|W(s)|}{\sqrt{2 s L L s}} .
\end{aligned}
$$

Moreover, for each $n \geqslant m$,

$$
\left\|\widetilde{Z}_{n} \mathbb{I}_{(m, \infty)}\right\|_{w} \leqslant \sup _{m \leqslant t \leqslant n} \sqrt{t} w(t) \sup _{m n \leqslant s<\infty} \frac{|W(s)|}{\sqrt{2 s L L \sqrt{s}}} .
$$

Hence, by Khintchin's law of the iterated logarithm for a Brownian motion, for each $m \geqslant 1$, we get

$$
\varlimsup_{n \rightarrow \infty}\left\|\tilde{Z}_{n} \mathbb{I}_{T \backslash \Delta_{m}}\right\|_{w} \leqslant \sup _{t \in T \backslash \Delta_{m}} \sqrt{t} w(t) \quad \text { a.s. }
$$

By Cauchy-Schwarz inequality, for each $\varphi \in \mathcal{S}$, it follows that

$$
\left\|\varphi \mathbb{I}_{T \backslash \Delta_{m}}\right\|_{w} \leqslant \sup _{t \in T \backslash \Delta_{m}} \sqrt{t} w(t) .
$$

Hence, by (1.3), (3.3), (3.4), and (3.5), we will have shown (3.1) as soon as we have proved that, for any given $\varepsilon>0$,

$$
\mathbf{P}\left(\left\{d_{w^{m}}\left(Z_{n}, \mathcal{S}\right)>\varepsilon \text { i.o. as } n \rightarrow \infty\right\}\right)=0
$$

for all sufficiently large $m$. This will be done once we have shown that for any given $\varepsilon>0$ there exists a real number $d(\varepsilon)>1$ such that

$$
\mathbf{P}\left\{\sup _{d^{k-1} \leqslant n<d^{k}}\left\|W\left(d^{k} \cdot\right)-W(n \cdot)\right\|_{w^{m}}>\frac{\varepsilon}{3} b\left(d^{k-1}\right) \text { i.o. as } k \rightarrow \infty\right\}=0
$$

for every $d \in(1, d(\varepsilon))$ and for all $m \geqslant 1$, and there exists an integer $m(\varepsilon) \geqslant 1$ such that

$$
\mathbf{P}\left\{d_{w^{m}}\left(Z_{d^{k}}, \mathcal{S}\right)>\frac{\varepsilon}{3} \text { i.o. as } k \rightarrow \infty\right\}=0
$$

for every $d>1$ and for all $m \geqslant m(\varepsilon)$. Indeed, given $\varepsilon>0$ put

$$
d(\varepsilon):=1+\frac{\varepsilon^{2}}{18\|\sqrt{I}\|_{w}^{2}} .
$$

Then take any real number $d$ such that

$$
1<d<\left(1+\frac{\varepsilon / 3}{\varepsilon / 3+\|\sqrt{I}\|_{w}}\right) \wedge d(\varepsilon)
$$


and choose an integer $k_{1}(d)$ such that, for all $k \geqslant k_{1}(d)$,

$$
1 \leqslant \frac{b\left(d^{k}\right)}{b\left(d^{k-1}\right)}<d
$$

By (3.7) and (3.8), for almost all $\omega$, there exists an integer $k_{2}(\omega)$ such that

$$
d_{w^{m}}\left(Z_{d^{k}}, S\right) \leqslant \frac{\varepsilon}{3} \quad \text { and } \quad \sup _{d^{k-1} \leqslant n<d^{k}}\left\|W\left(d^{k} \cdot\right)-W(n \cdot)\right\|_{w^{m}} \leqslant \frac{\varepsilon}{3} b\left(d^{k-1}\right)
$$

for all $k \geqslant k_{2}(\omega)$ and for all $m \geqslant m(\varepsilon)$. Moreover, by Cauchy-Schwarz inequality, for each $\varphi \in \mathcal{S}$, we get $\|\varphi\|_{w^{m}} \leqslant\|\sqrt{I}\|_{w}$. Thus, for every $n \geqslant$ $d^{k_{1}(d) \vee k_{2}(\omega)-1}$ and for all $m \geqslant m(\varepsilon)$, it follows from the preceding that

$$
\begin{aligned}
d_{w^{m}}\left(Z_{n}, \mathcal{S}\right) \leqslant & d_{w^{m}}\left(Z_{d^{k}}, \mathcal{S}\right)+\sup _{d^{k-1} \leqslant n<d^{k}} \frac{\left\|W\left(d^{k} \cdot\right)-W(n \cdot)\right\|_{w^{m}}}{b\left(d^{k-1}\right)} \\
& +\left[\frac{b\left(d^{k}\right)}{b\left(d^{k-1}\right)}-1\right]\left\|Z_{d^{k}}\right\|_{w^{m}} \\
& \leqslant \frac{\varepsilon}{3}+\frac{\varepsilon}{3}+(d-1)\left(\frac{\varepsilon}{3}+\|\sqrt{I}\|_{w}\right) \leqslant \varepsilon
\end{aligned}
$$

for some integer $k$ such that $d^{k-1} \leqslant n<d^{k}$. Hence, (3.6) follows from (3.7) and (3.8).

The proof of (3.7) and (3.8) is based on the following exponential inequality for supremum of an almost surely bounded mean zero Gaussian process $G=\{G(t): t \in D\}$ (cf. Lemma 3.1 in [11])

$$
\mathbf{P}\left\{\sup _{t \in D}|G(t)|>x\right\} \leqslant \exp \left\{-\frac{x^{2}}{4 \sup _{t \in D} E G^{2}(t)}\right\}
$$

for all sufficiently large $x$ (depending on a median of $\sup _{t \in D}|G(t)|$ ). Turning to the proof of (3.8), given $\varepsilon>0$, by the scale invariance of a Brownian motion and by $(3.10)$, we get

$$
\begin{aligned}
& \mathbf{P}\left\{\sup _{d^{k-1} \leqslant n<d^{k}} \sup _{t \in \Delta_{m}}\left|W\left(d^{k} t\right)-W(n t)\right| w(t)>\frac{\varepsilon}{3} b\left(d^{k-1}\right)\right\} \\
& \quad \leqslant \mathbf{P}\left\{\sup _{1 / d \leqslant r<1} \sup _{t \in \Delta_{m}}|W(t)-W(r t)| w(t)>\frac{\varepsilon}{3} \sqrt{2 L L\left(d^{k-1}\right) / d}\right\} \\
& \quad \leqslant \exp \left\{-\frac{\varepsilon^{2} L L\left(d^{k-1}\right)}{18(d-1)\|\sqrt{I}\|_{w}^{2}}\right\}
\end{aligned}
$$

for all sufficiently large $k$ (depending on $m$ ). Then, by the Borel-Cantelli lemma, (3.7) holds true for every $d \in(1, d(\varepsilon))$ with $d(\varepsilon)$ given. by (3.9) and for all $m \geqslant 1$. 
Turning to the proof of (3.8), fix an $\varepsilon>0$ again and define a stochastic process $\eta_{n}=\left\{\eta_{n}(t): t \in(0, \infty)\right\}$ by linearly interpolating the points $Z_{n}(i / m)$ at $i / m, i=0,1, \ldots, m^{2}$, and by letting equal to $Z_{n}(m)$ on $[m, \infty)$. Then, for each $r>0$,

$$
\begin{gathered}
\mathbf{P}\left\{\int_{0}^{\infty}\left(\dot{\eta}_{n}(u)\right)^{2} d u>r^{2}\right\}=\mathbf{P}\left\{\chi_{m^{2}}^{2}>2 r^{2} L L n\right\} \\
=\frac{1}{\Gamma\left(m^{2} / 2\right)} \int_{r^{2} L L n}^{\infty} x^{m^{2} / 2-1} e^{-x} d x \\
\sim \frac{\left(r^{2} L L n\right)^{m^{2} / 2-1} e^{-r^{2} L L n}}{\Gamma\left(m^{2} / 2\right)}, \text { as } n \rightarrow \infty .
\end{gathered}
$$

From here, using (3.10), we get

$$
\begin{aligned}
& \mathbf{P}\left\{d_{w^{m}}\left(Z_{d^{k}}, \mathcal{S}\right)>\frac{\varepsilon}{3}\right\} \leqslant \mathbf{P}\left\{\sup _{t \in \Delta_{m}}\left|\frac{\eta_{d^{k}}(t)}{r}-Z_{d^{k}}(t)\right| w(t)>\frac{\varepsilon}{3}\right\} \\
& +\mathbf{P}\left\{\frac{\eta_{d^{k}}}{r} \notin \mathcal{S}\right\} \\
& =\mathbf{P}\left\{\max _{2 \leqslant i \leqslant m^{2}} \sup _{t \in[(i-1) / m, i / m]} \mid \frac{1}{r}\left[W\left(d^{k} \frac{i-1}{m}\right)+[m t-(i-1)]\right.\right. \\
& \left.\left.\times\left[W\left(d^{k} \frac{i}{m}\right)-W\left(d^{k} \frac{i-1}{m}\right)\right]\right]-W\left(d^{k} t\right) \mid \frac{w(t)}{\sqrt{d^{k}}}>\frac{\varepsilon}{3} \sqrt{2 L L d^{k}}\right\} \\
& +\mathbf{P}\left\{\int_{0}^{\infty}\left(\dot{\eta}_{d^{k}}(u)\right)^{2} d u>r^{2}\right\} \\
& \leqslant c(m)\left(r^{2} L L\left(d^{k}\right)\right)^{m^{2} / 2-1} \exp \left\{-r^{2} L L\left(d^{k}\right)\right\}+\exp \left\{-\frac{\varepsilon^{2} L L\left(d^{k}\right)}{18 A(r, m)}\right\}
\end{aligned}
$$

for all sufficiently large $k$ (depending on $m$ and $r$ ), where

$$
A(r, m):=2\left(\frac{1}{r-1}\right)^{2} \sup _{t} t w^{2}(t)+\left(\sup _{t \notin\left[t_{1}, t_{2}\right]} t w^{2}(t)\right) \bigvee\left(\sup _{t \in\left[t_{1}, t_{2}\right]} \frac{w^{2}(t)}{m}\right)
$$

and $0<t_{1}<t_{2}<\infty$. By choosing $t_{1}, t_{2}, m$ and $r$ appropriately, one can make $18 A(r, m)$ to be less than $\varepsilon^{2} / 2$ say. So the Borel-Cantelli lemma yields (3.8). Then, (3.1) has now been proved.

To prove (3.2), by (3.4) and (3.5), we need only show that for each $m \geqslant 1$ and for all $\varphi \in \mathcal{S}$

$$
\begin{aligned}
\liminf _{n \rightarrow \infty}\left\|\tilde{Z}_{n}-\varphi\right\|_{w^{m}} & \leqslant \sup _{t \in \Delta_{m}} w(t)(1+t) \varlimsup_{n \rightarrow \infty} \sup _{t \in \Delta_{m}} \frac{1}{1+t}\left|Z_{n}(t)-\varphi(t)\right| \\
& =0 \text { a.s. }
\end{aligned}
$$

But this is already proved in the proof of the part (ii) of Theorem 1.4.1 in [7]. 
Here is the main result of the paper.

Corollary. Let $w$ be a weight function on $(0,1]$. If

$$
\lim _{t \downarrow 0} \sqrt{t} w(t)=0
$$

then the sequence $\left\{(2 n L L n)^{-1 / 2} S(n \cdot): n \geqslant 1\right\}$ almost surely clusters in $\mathbf{B}((0,1], w)$ to the Strassen set $\mathcal{S}$, that is we have

$$
\begin{cases}\lim _{n \rightarrow \infty} d_{w}\left(S_{n}, \mathcal{S}\right)=0 & \text { a.s. } \\ C_{w}\left(\left\{S_{n}: n \geqslant 1\right\}\right)=\mathcal{S} & \text { a.s. }\end{cases}
$$

P r.o of. By Theorem 2 of [14], one can redefine a Brownian motion $W$ and a partial sum process $S$ on a common probability space without changing their respective laws, in such a way that

$$
\lim _{t \rightarrow \infty}(2 t L L t)^{-1 / 2}|S(t)-W(t)|=0 \quad \text { a.s. }
$$

Moreover, for any $\delta>0$ and for each integer $n$, we have

$$
\begin{aligned}
& (2 n L L n)^{-1 / 2}\left\|S(n \cdot)-W(n \cdot) \mathbb{I}_{[1 / n, 1]}\right\|_{w} \\
& \quad \leqslant \sup _{t \leqslant \delta} \sqrt{t} w(t) \sup _{t \geqslant 1} \frac{|S(t)-W(t)|}{\sqrt{2 t L L t}}+\sup _{\delta \leqslant t \leqslant 1} w(t) \sup _{t \geqslant \delta n} \frac{|S(t)-W(t)|}{\sqrt{2 t L L t}} .
\end{aligned}
$$

Since $\delta$ is arbitrary and (3.11) holds, this in conjunction with (3.12) imply that

$$
\lim _{n \rightarrow \infty}\left\|S_{n}-\widetilde{Z}_{n}\right\|_{w}=0 \text { a.s. }
$$

and the result follows from Theorem.

Acknowledgement. I wish to thank Vidmantas Bentkus and David M. Mason for valuable remarks.

\section{REFERENCES}

1. Alexander $K$., Talagrand $M$. The law of the iterated logarithm for empirical processes on Vapnik-Červonenkis classes. - J. Multivar. Anal., 1989, v. 30, p. 155-166.

2. Baldi $P$., Ben Arous $G$., Kerkyacharian G. Large deviations and Strassen theorem in Hölder norm. - Stoch. Process. Appl., 1992, v. 42, p. 171-180.

3. Beibel M.; Lerche $H$. R. A refined large deviation principle for Brownian motion and its applications to boundary crossing. - Stoch. Process. Appl., 1994, v. 51, p. 269276.

4. Ben Arous G., Ledoux M. Schilder's large deviation principle without topology. - In: Asymptotic Problems in Probability Theory: Wiener Functionals and Asymptotics, (Proc. Taneguchi Intern. Symp., Sanda \& Kyoto 1990, Pitman Research Notes in Math., Series 284.) Ed. by K. D. Elworthy and N. Ikeda. Longman, 1993, p. 107-121. 
5. Chatterji S. D. Factorization of positive finite operator-valued kernels. - In: Prediction Theory and Harmonic Analysis: The Pesi Masani Volume./Ed. by V. Mandrekar and H. Salchi. Amsterdam: North-Holland, 1983, p. 23-36.

6. Deheuvels $P$., Lifshits $M$. A. Strassen-type functional laws for strong topologies. Probab. Theory Relat. Fields, 1993, v. 97, p. 151-167.

7. Deuschel J.-D., Stroock D. W. Large Deviations. Boston: Academic Press, 1989.

8. Dunford N., Schwartz J. T. Linear Operators. Part I: General Theory. New York: Interscience Publishers, 1967.

9. Khintchine $A$. Sur la croissance locale des processes stochastiques homogénes á accroissements indépendantes. Bulletin de l'Académie des Sciences de l'URSS, Serie mathematique, 1939, v. 5-6, p. 487-508. (In Russian.)

10. Lai T. L. Reproducing kernel Hilbert spaces and the law of the iterated logarithm for Gaussian processes. - Z. Wahrscheinlichkeitstheor. verw. Geb., 1974, B. 29, S. 7-19.

11. Ledoux M., Talagrand M. Probability in Banach Spaces. Berlin-Heidelberg: SpringerVerlag, 1991.

12. Nachbin L. Elements of Approximation Theory. (Math. Studies 14.) Princeton: Van Nostrand, 1967.

13. Nisio $M$. On extreme values of Gaussian processes. - Osaka J. Math., 1967, v. 4, p. 313-326.

14. Strassen $V$. An invariance principle for the law of the iterated logarithm. - Z. Wahrscheinlichkeitstheor. verw. Geb., 1964, B. 3, S. 211-226.

15. Suchanecki $Z$. Cylindrical measures and cylindrical processes on locally convex spaces. - Probab. Math. Statist., 1981, v. 2, p. 71-81.

16. Wichura $M$. J. Functional laws of the iterated logarithm for the partial sums of i.i.d. random variables in the domain of attraction of a completely asymmetric stable law. - Ann. Probab., 1974, v. 2, p. 1108-1138.

Поступила в редакцию 10.III.1994

Исправленный вариант

12.V.1996 